\title{
Touch for Health: Use of Pavlovian Processes with Physical Touch as a Means to Improve Menstrual Hygiene Management Initiatives, Measured by Willingness to Pay
}

\author{
Siobhan K. Yilmaz ${ }^{1}$ - Alok K. Bohara' ${ }^{1}$ Soumi Roy Chowdhury²
}

Published online: 23 July 2019

(c) The Author(s) 2019

\begin{abstract}
Background To combat the lack of proper facilities and menstrual health knowledge in developing countries, many WASH (Water, Hygiene, and Sanitation) initiatives are including menstrual hygiene management (MHM) components. However, evidence shows that prior efforts have not been ultimately successful in inducing relevant behavior changes, due in part to cultural constraints and unidimensional interventions. As such, MHM research may need to include consideration of new theories/approaches. Evidence is growing of the role that physical presence/proximity and ability to touch objects has on incentives for consumers to purchase goods, captured through willingness to pay (WTP) figures. Such findings can be partially explained by Pavlovian processes.

Objective This study sought to provide field-work validation of such findings of the role of Pavlovian processes and endowment effect on WTP figures for a female hygiene kit, reflecting motivation to adopt better hygiene behaviors.

Methods This study used primary survey data collected from females in two upper-level schools in southwestern Nepal $(n=169)$. When presented in conjunction with a hygiene education session, one group of females was allowed physical interaction with a female hygiene kit, while another was not, before being surveyed on their WTP for the kit. Both non-parametric and parametric statistical analyses were performed to assess the impact of this ability to touch the kits on WTP figures.

Results Results show a statistically significant difference between the WTP figures of those females allowed interaction with the kits prior to being surveyed and those who did not. This confirms the positive impact of physical presence/touch on motivations to use/acquire hygiene-related tools.

Conclusions Such findings reveal how, through application of the theory of Pavlovian processes, future (menstrual) health education efforts could harness the human instinct to consume, remember, and use those objects presented in physical form, and include personal contact and demonstrations of better health practices in future MHM and WASH education initiatives. Such an approach may allow protocols and interventions to have more success, and dissemination of healthier behaviors and knowledge to be more prominent.
\end{abstract}

\section{Introduction}

Electronic supplementary material The online version of this article (https://doi.org/10.1007/s41669-019-0168-6) contains supplementary material, which is available to authorized users.

Siobhan K. Yilmaz

yilmazsiobhan@gmail.com

Alok K. Bohara

yilmazsiobhan@gmail.com

Soumi Roy Chowdhury

yilmazsiobhan@gmail.com

1 Department of Economics, University of New Mexico, Albuquerque, NM, USA

2 National Council of Applied Economic Research (NCAER), New Delhi, India
Menstrual hygiene management (MHM) is becoming an important sub-area of focus for water, sanitation, and hygiene (WASH) researchers [1], where many small-scale projects point to lack of access to adequate information, products, and disposal systems within many low- and middle-income countries (LMICs) [2]. Existing evidence shows that ignoring the impacts of MHM can be quite detrimental to the overall growth and success of women, with inadequate menstrual hygiene linked to reproductive tract infections [3, 4], school attendance/dropping out [5-10], and impacts on overall psychosocial well-being [11-14]. The lack of proper facilities and education in many countries demonstrates the 


\section{Key Points for Decision Makers}

Higher willingness to pay (WTP) for a feminine hygiene kit was found among young Nepali females allowed physical contact with the product itself, prior to being asked their WTP.

Results show promise of including physical contact and demonstrations of better health practices and technology in future menstrual health management and water, hygiene, and sanitation education initiatives.

necessity for policies improving the quality and availability of sanitation and hygiene options [15].

One of the common barriers to such aims among WASH initiatives is a frequent lack of consideration of women and girls in sanitation policy due to the stigma often surrounding menstruation [2]. Such stigma is exemplified by how education about menstruation itself is often decoupled from sex education in the developing world due to the cultural sensitivities of the topic [10]. The burden has fallen on academic researchers and non-governmental organizations (NGOs) to push for improved knowledge and sanitation-supporting infrastructure in the developing world, where the best approach is often through school-based interventions [11].

MHM interventions found in the literature can primarily be segmented into those supplying 'hardware' or 'software' materials to resource-poor countries [16]. Hardware-type interventions are those aimed at reducing material deprivations by offering free or subsidized sanitary products [17-20], or by improving sanitary/hygiene infrastructure at schools [21]. On the other hand, software-type interventions are aimed at improving hygiene knowledge and management deficits through educational initiatives [13, 22-24]. Recent systematic reviews have generally agreed that there are localized, modest achievements in hygiene awareness and practices from software-based MHM interventions, but uptake of sanitary products themselves (hardware-based approaches) has often been hard to measure, or has been low $[16,25]$. Such findings are consistent with the prevailing trend showing that currently implemented interventions are not ultimately as successful as anticipated.

The level of awareness on menstrual (and reproductive) hygiene is still generally low across much of the developing world. Surveys have shown that there is still early initiation of sexual activity, an often involuntary context for intercourse, presence of high-risk behaviors, and inadequate levels of knowledge about protection from and transmission of sexually transmitted infections [26]. Furthermore, there is evidence of a lack of proper hygiene knowledge and behaviors, even among health professionals [27-30]. In addition to continuing the lack of information, attempts to alleviate this constraint are still met with the reality that often the health behaviors of even informed people do not change [17-19, 31].

The failure of currently implemented MHM interventions may be due in part to the underlying cultural environment that makes change hard. There is generally a lack of support from teachers (who are often male), there is teasing by peers (often boys, ill-informed of the processes), poor familial support, and lack of supporting infrastructure (both at home and school), all fed by the cultural taboo/stigma often surrounding menstruation [25]. This situation has led to a call for rethinking the way MHM education is imparted. There is a need to build/find new best practices in (menstrual) health education, with perhaps a more universally applicable approach, not so hindered by individual culture. Additionally, many interventions have remained unidimensional, with only a hardware or software approach. Hennegan and Montgomery [16] recommend that future MHM research begin exploring combinations of hardware and software approaches, with innovative techniques to better capture an individual's preferences.

The measurement of preferences and motivation to adhere to new health-related behaviors has often been measured via willingness to pay (WTP) estimates [32-34]. We follow this methodology, analyzing a new potential 'best practice' in relation to (menstrual) health education/technology adoption. We implement work from psychology literature on Pavlovian processes and physical touch, leveraging research showing that consummatory responses are less frequently triggered in purely hypothetical situations, and that ownership feelings increase from physical interactions with objects of interest (more details can be found in the Background section of this paper). There is growing awareness across disciplines of the multitude of ways that learning impacts behavior, including how environmental cues may play a key role [35]. The processes examined in this study apply some of these findings in a real-world setting, where our results provide impetus for new approaches to (menstrual) health education, particularly in the developing world.

We utilized a treatment-control type framework to see if physical touch increases WTP for a feminine hygiene kit, presented in conjunction with a feminine hygiene education session. In investigating the differences in WTP between two groups exposed to similar hygiene sessions, our hypothesis was that having more contact with the kits would increase WTP. Such a finding would be a strong indicator of the benefits of including personal contact and demonstrations of better health practices in future menstrual health management (MHM) and WASH education initiatives. 


\section{Background}

In the economics literature investigating decision making and consumer adoption, there is a growing emphasis on using theories from other social sciences such as psychology [36, 37]. Applications of psychology-based theories have appeared in both methodological survey development and experimentation research focused on the decision-making patterns of individuals.

Bushong et al. [38] formally introduced the psychological process of conditioned responses to economic evaluations. Through a series of laboratory experiments comparing WTP estimates for food items using written, pictorial, or physical form representations, researchers determined that physical presence of the items elicited the highest WTP/bids. Ultimately, authors attributed this finding to that of Pavlovian processes.

In the language of psychology, the physical presence of an item serves as an unconditioned stimulus (US) triggering consummatory responses. Unless there is another (nonphysical) cue that has been associated with this response long enough to form a conditioned stimulus (CS), the consummatory response will not occur in the absence of the physical presence of the item. Authors theorized that the names and pictures of food (and other items such as mugs and DVDs) are not associated with the consumer goods frequently enough for these virtual presentation mediums to serve as a CS. Novel to the work of Bushong et al. [38] was that their results ran contrary to typical economic arguments that higher WTP results can be explained based primarily on improved information and less uncertainty (the largest gain in information is actually from text to picture). Additionally, the magnitude of WTP effects for goods presented in physically rather than through virtual representation was found to be similar for familiar and unfamiliar items.

The literature also shows that incorporating the ability to touch items further enhances WTP. There is evidence that physical contact with products allows consumers to become more committed to using them. In a follow-up to the work of Bushong et al. [38], researchers re-evaluated the prior findings by including the element of tactile (e.g. touch) sensation, by giving subjects the ability to handle both appetitive (e.g. food/drink) and non-appetitive items [39]. Researchers found higher mean purchase rates among real versus pictorial image conditions, regardless of product type.

In their investigation of the distinction between factual and subjective feelings of ownership, Reb and Connolly [40] focus on the endowment effect consumers give to items that they possess (e.g. ascribing more value to things merely because they own them). They found that it is physical possession, not factual ownership, that can increase the personal monetary valuation of an object. They relate this finding to the principle of loss aversion, wherein loss of something is often weighted greater than the gain of the same thing [41]. Taking these findings even further, Wolf et al. [42] were able to provide evidence of the length of ownership effect, through mere touch, using an experimental bidding for mugs. They had hypothesized that duration of exposure to an item fosters increased attachment, and thus valuation, because partial adaptation to ownership begins immediately following initiation of physical contact. Participants in their experiment were shown to bid higher amounts when they were allowed physical contact with a mug for 30 s versus only 10 s.

Why mere touch appears to increase perceived ownership is believed to be closely associated with increased affective reactions [43]. Some researchers posit that there is a greater emotional response to an object obtainable only through physical contact, which cannot be achieved via additional information on an object via descriptors. Romer [44] posits that decision mechanisms can be segregated into those based on thoughts and those based on feelings, where a thinking-based 'mistake' can be altered in the face of new information, while a feeling-based 'mistake' will not. Feeling-based mechanisms are therefore more likely to be specialized in terms of the type of information that they can process, and much less responsive to the types of information conveyed by language. Hence, physical touch adheres to a feelings-based mechanism more so than a thoughts-based mechanism.

Thus, the literature has provided evidence of the connection between the consummatory response, proximity, and motivation to acquire, with pathways that are inherently founded in Pavlovian thinking. However, literature focused on health education and best practices has not yet begun to incorporate these ideas.

\section{Methods}

\subsection{Data and Variables}

This study makes use of primary survey data collected by the Pratiman-Neema Memorial Foundation (PNMF) from Nepal in May 2016. Women2Be, a non-profit organization based out of Albuquerque, NM, provided female hygiene packets, in the style of those put together by Days for Girls, in conjunction with the Nepal Study Center of the University of New Mexico. These kits included two pairs of underwear, eight liners, two shields, a washcloth, soap, Ziploc bags for washing/storage, and a fabric bag to hold all components. These kits are made of colorful fabrics so as to not automatically label them as being for the purpose of menstruation, and are quick-drying and require little water for cleaning. Along with providing girls and women these reusable kits, 
lasting for up to 3 years, the director of Women2Be and supporting staff provided educational information about female health and hygiene, and a survey was administered to various groups of women/girls. ${ }^{1}$

The analysis from this paper uses data from females surveyed in two schools in Bhairahawa-Pratiman-Neema Memorial Health Institute (PNMHI) and Paklihawa. At the conclusion of the hygiene talk, each group was given a paper survey (translated from English into Nepalese and available in the Electronic Supplementary Material) to fill out on topics including perceptions towards female hygiene, cultural and social norms surrounding menstruation, school absences, hygiene facilities available at school, demographic information, and, critical to this study, an open-ended question on WTP for the kit, framed as "What is the maximum amount you would pay for this reusable sanitary pad packet?" The question was preceded with two written paragraphs detailing the importance of proper feminine hygiene practices to prevent infections, and promoting this kit as a means to potentially improve quality of life and reduce school absences. These paragraphs served as a primer reinforcing what was presented to the girls in the hygiene talk.

Those at PNMHI (our 'treatment') school were given the kit to examine, touch, and personally interact with, before being asked to fill out the survey. Paklihawa (our 'control') school was not presented with the kits to physically touch and examine prior to completing their survey and giving their WTP. At the conclusion of both sessions, each participant received their own feminine hygiene kit to take home, but neither school group was informed that they were able to keep the kits until after the surveys were completed.

Those allowed physical interaction would be expected to have a larger endowment effect and Pavlovian response to the health-related technology they were being asked to adopt. Consistent with those findings from literature, we would expect higher WTP estimates from the treatment group of girls. Formally:

Hypothesis 1 Females presented with the kits before survey will exhibit a higher WTP for the reusable female hygiene kit than those not presented the kits before surveying.

Initial examination of the distribution of raw WTP across the two groups was non-normal, and an attempt was made to better normalize this via a log-normal approach. This full

\footnotetext{
${ }^{1}$ Informed consent was sought in administration of the survey and was approved by local authorities. Parents and teachers were present at this event and representatives of the Pratiman-Neema College were on hand to ensure that the study was carried out in a manner consistent with the ethical standards of the Declaration of Helsinki.
}

empirical specification is shown below. The dependent variable, $\ln (W T P$ ) is the natural log of WTP (given in Nepalese Rupees). Treat was our key treatment (kit before survey) dummy (e.g. a binary variable coded as 1 for presence and 0 for non-presence). Age and AgeMens represent the age of the female currently, and the age of the female when she first started menstruating (presented in bins), respectively. Hyg is a vector of current hygiene product usage dummies, and Env is a vector of environmental indices. $X$ represents additional demographic controls for religion, caste, and wealth indicators. Finally, $\varepsilon$ represents a white-noise error term

$$
\begin{aligned}
\ln (\text { WTP })_{i}= & \beta_{0}+\beta_{1} \text { Treat }_{i}+\beta_{2} \text { Age }_{i}+\beta_{3} \text { AgeMens }_{i} \\
& +\beta_{4} \text { Hyg }_{i}+\beta_{5} \text { Env }_{i}+\beta_{6} X_{i}+\varepsilon_{i}
\end{aligned}
$$

We accounted for age, as well as the age of first menstruation, under the assumption that older women will be better able to pay for a new hygiene kit, while those women who have been menstruating longer may have had more time to develop a preference for one product type over another. Additionally, older women may become more motivated to try a new product, having already explored existing options. The hygiene product type is accounted for with binary variables representing disposable and reusable hygiene products, with the omitted category as old rags/clothes.

We also included environmental indices to control for home and school life, anticipated to influence the desirability of the product. The index representing school environment consists of the presence/absence of certain key hygiene facilities/ infrastructure at school. The index for the home environment captures the behavioral restrictions/taboos girls are subject to during their menstruation. We utilized principle component analysis, a statistical data reduction technique, of the various factors to create these indices, relying on the first principal component, which is the summary variable capturing the most variance. Uncertainty in direction of influence led us to not make a specific hypothesis about the direction of influence on WTP. For example, the availability of hygiene products, perhaps at school, could negatively impact WTP if girls see less need for additional support. Alternatively, there could be a desire to have more infrastructure at school to make attendance easier. Similarly, if there are lots of social pressures/cultural taboos surrounding menstruation, girls may seek to better manage it to appear cleaner, thus increasing WTP. However, if taboos are engrained and strong enough, girls could also see less need for hygiene kits, reflected in a lower WTP.

To account for missing data for our dependent variable measure, we used the mean value for each school group. Due to missing data for three observations on the age at which females started menses, we chose to remove these observations. The total estimable sample was 169 observations, of which 73 were in the treatment group and 96 were in the control group. 


\subsection{Non-Parametric}

The highly skewed nature of our primary data, and differences found across descriptive statistics, led us to perform non-parametric testing, in addition to regression estimation, to compare mean and distributional differences. To compare means, we used the Wilcoxon signed-rank test (Mann-Whitney), which is an alternative to the parametric, paired Student's $t$ test, in the face of non-normal distributions. This test is used to determine if two dependent samples were selected from a population having the same distribution-in our case, do our samples have the same distribution of WTP? Additionally, we performed the Kolmogorov-Smirnov test, which is another means by which one can test if two data samples come from the same distribution (although no claim is made as to whether or not this underlying distribution is normal). Finally, Fisher's exact test, commonly used in studies with small sample sizes, was used as a comparison of median WTP values, testing if the samples were drawn from populations with the same median.

\subsection{Regression Estimation}

Given the continuous nature of our dependent variable and its highly skewed nature, the initial regression estimation was log-linear, beginning with a $t$ test or treatment-only version. We sequentially incorporated additional controls, while maintaining the log-linear approach. The addition of these extra controls was done to determine if they affected the significance of our initial findings due to the multiple differences between our control and treatment groups. Given such evidence that girls were different along multiple dimensions, including percentage Dalit, average wealth index, average age, and type of hygiene product currently being used, we also ran our log-linear models incorporating inverse probability weighting (IPW). This is a statistical technique enabling us to estimate the potential outcome if all subjects in our study were assigned either group, and removing some of the bias of the two groups being different along certain demographic lines.

To provide further robustness, we attempted to correct for the highly skewed nature of our data, using a non-linear gamma approach (generalized linear model [GLM]), with a log link function, where we again accounted for differences via IPW. We also ran our most preferred models with bootstrapped standard errors $(n=500)$. Finally, we checked for outliers by examining the distribution of residuals from each best-fit model and found no extreme outliers beyond two standard deviations (both log-linear and GLM approaches).

\subsection{Sensitivity Analysis: Larger Sample}

To explore the sensitivity of our findings, we have also included in the Electronic Supplementary Material the results of repeating our non-parametric testing and regression estimation with a larger sample size $(n=307)$. In December 2017, an additional 138 young women were surveyed in the central hills area of Purkot, Nepal. This group was surveyed in a manner consistent with the control group (e.g. Paklihawa) from May 2016, i.e. no interaction with the kit before a WTP query. We chose not to include this extended control group in the primary analysis presented in this paper due to the time lag between data collection and the associated potential unknown biases caused by this situation; however, we feel it serves as a useful check of our original findings.

\section{Results}

\subsection{Descriptive Statistics}

Initial observations show that the treatment group showed higher mean WTP for the kit than the control group: 375.76 vs. 301.50 Rupees. Figure 1 provides additional visual evidence of a pattern of increased WTP among the treatment group, both in terms of the probability density (PDF) and cumulative density (CDF) of raw WTP figures. Comparison of the two study groups across other key demographic and explanatory variables can be found in Table 1 .

The average age of those in the treatment group was 19.4 years, and 16.2 years for the control group; however, the range for the control group of women was much larger. There is, though, a similar mean value for age of first menses across the two groups, of 12-13 years. It also appears that the two groups differ in their current hygiene practices, with the treatment group currently relying more on disposable products. The groups do appear to differ in certain school infrastructure and cultural limitation factors surrounding menstruation; however, both groups express discontent with their lives during menstruation, with $58.1 \%$ (control) and $98.6 \%$ (treatment) reporting that it is difficult to manage life during their menstrual cycle. There is a statistically significant difference in the size of the Dalit (lower caste) population in the control group (23\%) versus the treatment group (7\%), likely highly correlated with overall socioeconomic status (statistically higher average score on the wealth index).

\subsection{Non-Parametric}

Table 2 presents a summary of the significance of each of the non-parametric tests employed in our analysis. Results of the various non-parametric tests show statistically significant rejection of three noted null hypotheses that the control and treatment groups have the same WTP distributions, the same density functions (PDFs), and the same median WTP values. 
Fig. 1 Comparison of WTP distributions by treatment status

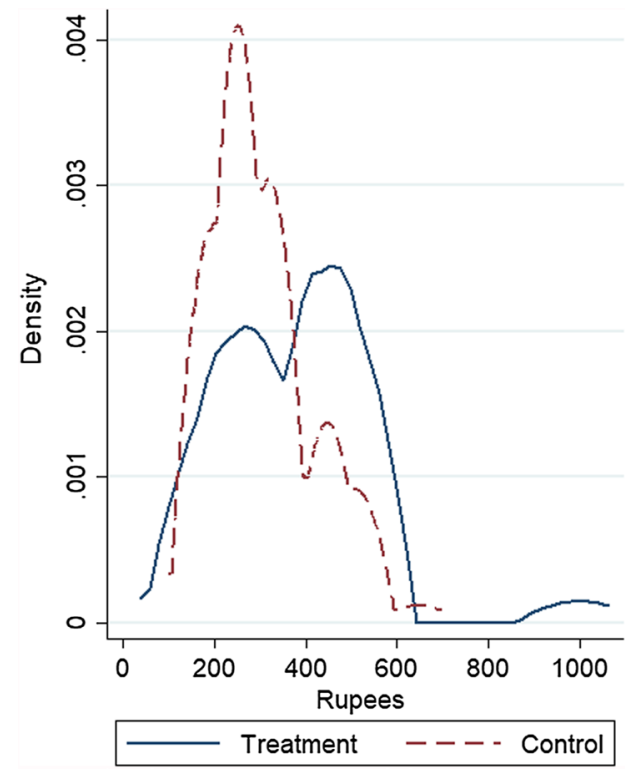

Thus, there is consistent evidence that the two groups of surveyed females elicit statistically different WTP estimates. The Wilcoxon rank-sum (or Mann-Whitney) test for equality of distributions also shows that that there is a $63 \%$ chance that a randomly selected individual from the treatment group would have a higher WTP than a randomly selected individual from the control group. Furthermore, re-examination of the CDFs (Fig. 1) also supports that those females in the treatment group exhibited higher WTP, visualized through the higher CDF for the treatment group across most of the distribution. Finally, the kernel density plot for the treatment group is shifted right of that of the control group (towards higher WTP values).

\subsection{Regression Estimation}

Table 3 presents a summary of results from each of the four estimation methods employed (log-linear and GLM, both with and without IPW), reflecting the best-fit model under each estimation approach, based on the minimum Akaike Information Criterion (AIC). The AIC deals with the trade-off between the goodness of fit of the model and the simplicity of the model [45]. Appendix Tables 4, 5, 6 and 7 show the detailed results of each model. As seen in Table 3, regardless of specification, there is at least marginal significance of the treatment effect. Note that significance is based on bootstrapped standard errors under scenarios without IPW, and robust standard errors under scenarios that do include IPW.

Examination of the detailed results (Tables 4, 5, 6, 7) reveal that the treatment effect within each preferred model indicates interaction with the kit elicits a $15.8-24.6 \%$ increase in WTP (log-linear approaches), or an 18.7-25.8\% increase in WTP (non-linear gamma with log-link). Greater significance is found through estimation based on the nonlinear gamma approach.

Another key conclusion from the detailed regression output tables is that there is a consistent positive effect of age on WTP, across all model and estimation strategies. As a woman becomes another year older, her WTP is increased approximately $1-2 \%$. There is also occasional significance of the dummies representing current use of either a reusable or disposable hygiene product, with a range of between a 21 and $28 \%$ decrease in WTP amounts.

\subsection{Sensitivity Analysis: Larger Sample}

In the Electronic Supplementary Material, Tables B1-B5 present the results of our sensitivity analysis with the expanded control group. Both non-parametric and regression results support our original findings that the ability to touch the kits prior to being asked WTP increased WTP estimates. Significance is found for all non-parametric tests, with the Wilcoxon rank-sum test for equality of distributions showing that there is a $65 \%$ chance that a randomly selected individual from the treatment group would have a higher WTP than a randomly selected individual from the control group. Using the lowest AIC, we again find significance across multiple specifications for the treatment variable, indicating a magnitude of a 19-34\% increase in WTP from physical contact with the feminine hygiene kits. 
Table 1 Descriptive statistics of variables

\begin{tabular}{|c|c|c|c|c|c|c|}
\hline \multirow[t]{2}{*}{ Variables } & \multirow[t]{2}{*}{ Description } & \multicolumn{2}{|c|}{ Control $[n=96]$} & \multicolumn{2}{|c|}{ Treatment $[n=73]$} & \multirow[t]{2}{*}{ Difference $^{\mathrm{a}}$} \\
\hline & & $\begin{array}{l}\text { Mean/per- } \\
\text { centage }\end{array}$ & SD & Mean & SD & \\
\hline \multicolumn{7}{|l|}{ Dependent variable } \\
\hline $\mathrm{WTP}^{\mathrm{b}}$ & $\begin{array}{l}\text { WTP for a feminine hygiene kit, in Nepalese } \\
\text { Rupees }\end{array}$ & 301.05 & 116.07 & 375.75 & 165.67 & $-74.70 * * *$ \\
\hline \multicolumn{7}{|l|}{ Explanatory variables } \\
\hline Age, years & Age of female & 16.24 & 6.012 & 19.40 & 2.13 & $-3.15 * * *$ \\
\hline Age at first menses & $\begin{array}{l}=1 \text { if age between } 10 \text { and } 12 \text { years; } 2 \text { if } \\
\text { age between } 13 \text { and } 15 \text { years; and } 3 \text { if age } \\
\text { between } 16 \text { and } 19 \text { years }\end{array}$ & 1.85 & 0.481 & 1.81 & 0.461 & 0.046 \\
\hline \multicolumn{7}{|l|}{ Hygiene product use ${ }^{c}$} \\
\hline Reusable & $\begin{array}{l}\text { Percentage of females currently using a reus- } \\
\text { able hygiene product }\end{array}$ & $18.8 \%$ & - & $6.8 \%$ & - & $11.9 * *$ \\
\hline Disposable & $\begin{array}{l}\text { Percentage of females currently using a dispos- } \\
\text { able hygiene product }\end{array}$ & $63.5 \%$ & - & $89 \%$ & - & $-25.4 * * *$ \\
\hline School environment & $\begin{array}{l}\text { Percentage of females reporting the presence of } \\
\text { the following items at school }\end{array}$ & & & & & \\
\hline Hygiene kit & & $32.3 \%$ & & $79.5 \%$ & & $-47 * * *$ \\
\hline Disposal bin & & $87.5 \%$ & & $72.6 \%$ & & $14.9 * *$ \\
\hline Soap & & $15.6 \%$ & & $82.2 \%$ & & $-66.6^{* * *}$ \\
\hline Separate toilet & & $31.3 \%$ & & $13.7 \%$ & & $17.6^{* * *}$ \\
\hline Curriculum & & $94.8 \%$ & & $86.3 \%$ & & $8^{*}$ \\
\hline Cultural norms & $\begin{array}{l}\text { Percentage of females reporting each of the fol- } \\
\text { lowing activities during menstruation }\end{array}$ & & & & & \\
\hline Forced isolation & & $7.3 \%$ & & $1.4 \%$ & & $5.9^{*}$ \\
\hline No cultural participation & & $86.5 \%$ & & $49.3 \%$ & & $37.1 * * *$ \\
\hline Not allowed in the kitchen & & $63.5 \%$ & & $56.2 \%$ & & 7.4 \\
\hline Not allowed to meet family & & $29.2 \%$ & & $26 \%$ & & 3.1 \\
\hline $\begin{array}{l}\text { Not allowed in the worship } \\
\text { area }\end{array}$ & & $96.9 \%$ & & $86.3 \%$ & & $10.6^{* *}$ \\
\hline \multicolumn{7}{|l|}{ Demographic controls } \\
\hline Buddhist & $\begin{array}{l}\text { Percentage of females belonging to the Bud- } \\
\text { dhist religion }\end{array}$ & $1.04 \%$ & - & $2.74 \%$ & - & -0.017 \\
\hline Dalit & $\begin{array}{l}\text { Percentage of females belonging to the Dalit } \\
\text { caste }\end{array}$ & $22.9 \%$ & - & $6.8 \%$ & - & $0.161 * * *$ \\
\hline Wealth index & $\begin{array}{l}\text { Sum of binary (1/0) self-reported yes answers } \\
\text { to self or family owning land and a cement } \\
\text { home }\end{array}$ & 1.58 & 0.610 & 1.81 & 0.490 & $-0.225^{* *}$ \\
\hline
\end{tabular}

$S D$ standard deviation, $W T P$ willingness to pay

a*** $p<0.01, * * p<0.05, * p<0.1$

${ }^{\mathrm{b}}$ Regressions are run using $\ln$ (WTP) as the dependent variable

'Omitted category is 'rags/old clothes'

\section{Discussion, Conclusions, and Policy Implications}

Bushong et al. [38] proposed and tested how Pavlovian processes lead to a higher WTP for an item when physically present. Our results confirm this through a field experiment of an actual hygiene-related product, where physical presence and contact with the technology appears to induce higher WTP estimates. Both non-parametric testing and regression analyses support our hypothesis across multiple specifications. Even when accounting for potentially nonrandom distribution of our samples across the two groups, IPW estimates produce even stronger estimates of our treatment coefficient.

We also find marginally significant results with regard to age, consistent with initial reasoning. As a female ages, 
Table 2 Non-parametric tests comparing means, medians, and distributions

\begin{tabular}{ll}
\hline Test & $\begin{array}{l}\text { Significance } \\
\text { of rejection }\end{array}$ \\
\hline $\begin{array}{l}\text { Wilcoxon rank-sum test }\left(\mathrm{H}_{0} \text { : control and treatment }\right. \\
\text { from the same distribution })\end{array}$ & $* * *$ \\
$\begin{array}{l}\text { Two-sample Kolmogorov-Smirnov test } \\
\left(\mathrm{H}_{0}: \text { control PDF = treatment PDF }\right)\end{array}$ & $* * *$ \\
$\begin{array}{l}\text { Fisher's exact test } \\
\left(\mathrm{H}_{0}: \text { median WTP control=median WTP treatment }\right)\end{array}$ & $* * *$ \\
\hline
\end{tabular}

$P D F$ probability density function, $W T P$ willingness to pay

a***p $<0.01, * * p<0.05, * p<0.1$

Table 3 Summary multivariable empirical results table

\begin{tabular}{llllll}
\hline Effect on WTP & \multicolumn{2}{l}{ Without IPW } & & & With IPW \\
\cline { 2 - 3 } \cline { 5 - 6 } & Log-linear & GLM & & Log-linear & GLM \\
\hline Treatment & $+*$ & $+^{* *}$ & & $+* * *$ & $+* * *$ \\
\hline
\end{tabular}

Each reported significance level is for the most preferred model under each specification, based on the minimum AIC from Tables 4, 5, 6 and 7

Bootstrapped standard errors used for models without IPW; robust standard errors used for models with IPW (bootstrapping does not allow for weights)

$I P W$ inverse probability weighting, WTP willingness to pay, $G L M$ generalized linear model, AIC Akaike Information Criterion

$* * * p<0.01, * * p<0.05, * p<0.1$

she is expected to have more income available, which can contribute to higher WTP figures; however, we control for wealth and therefore the impact we see is more of a pure age factor. With increased age, there is an associated increase in education, some of which may be health-related. Those older females observed are likely teachers within the school environment and would face similar restrictions/desires for enhanced menstrual hygiene support to girls more recently entering their menses, but the relevance of the hygiene information session and the importance of implementing better feminine hygiene behavior, including the use of a kit such as the one provided, may be better internalized by more mature females.

Our findings add to the literature examining the use of physical objects to elicit more behavior change adoption, where additional neurological and preliminary findings support such an application [46-48]. Neuroeconomists have determined that sensory and vivid information are seen as more likely to contribute to the decision process than the slower consideration of long-term and abstract consequences [49]. Plus, there is evidence that the full reward circuitry in the brain is not stimulated with imagined/ hypothetical situations [50]. Taken as a whole, the existing evidence points to a different reaction, biologically and psychologically, for humans when presented with a physical object (that they can touch). These differences are then manifested in higher valuations and willingness to adopt/ pay for technology [51]. This offers up a means by which to rethink (menstrual) health education, approaches to not only inform but also motivate changes in health-related behavior and technology adoption.

The apparent lack of success of prior intervention efforts in menstrual hygiene and sanitation across the developing world means that evidence of effective and diverse teaching tools may be quite policy relevant. That physical contact with the hygiene kits appears to induce higher WTP figures for girls in our study suggests a policy of incorporating more interactive materials in health/hygiene education throughout the developing world and exposing learners to the health technologies being promoted (in real-life). Despite some evidence that experiential learning in nutritional education may not impact children's willingness to taste unfamiliar vegetables [52], prior work in the developed world within other health education fields (i.e. oral hygiene and children's nutritional choices) has shown success incorporating real objects/experiential learning [53-56]. Additionally, there is evidence in weight-management research that the presence of physical food can undermine healthy food lessons that were originally presented in hypothetical situations $[57,58]$. It thus appears important in healthrelated education to incorporate real experiences/objects into the lessons themselves to increase the adoption of healthy behaviors.

In terms of study limitations, there is the potential for some bias in our estimates, given that the girls from the treatment group are different along multiple fronts, including that, on average, they report as being unhappier during their menstruation. This may lead them to be more intrinsically motivated to find an alternative solution to manage their menstruation. The hygiene kit may offer this solution, giving them some additional motivation to want to purchase the hygiene kit (e.g. a higher WTP). However, we control for cultural norms, many of which have been linked to depression/unhappiness [14, 49, 50], in our regressions, and significance remains through robustness checks. Additionally, we did consider the role of income through including a wealth index reflecting assets indicative of higher wealth, in certain models; however, their addition does not appear to add any additional strength to the models (see Tables 4, $5,6,7)$.

We also acknowledge that the significance we do find for our treatment variable is strongest only under certain conditions, namely with probability weighting. However, the use of probability weighting is to account for the likelihood of being a member of one treatment group over another. Thus, we sought to overcome some of the issues we note with 
regard to differences between the two treatment groups in using such weighting. Since the results are even stronger for our treatment variable under such conditions, we are encouraged that our results can usefully help inform future work and policy development.

Another element of WTP analyses not fully fleshed out in this study is that of consequentiality. To ensure realistic valuation answers, researchers often need to determine if surveys are perceived as potentially consequential to the respondents [59-62]. We did not directly test for consequentiality; however, it is not unreasonable to believe that the females surveyed would believe that that their WTP answers might inform policy makers in how to price or to even offer selling the feminine hygiene pack in the future. Additionally, we feel that these girls would genuinely care if this feminine hygiene kit were produced and marketed, given the primer paragraphs in our survey. Nevertheless, in follow-up work, we will consider including questions to capture the consequentiality of such WTP studies.

This work offers up good promise for future work in the fields of health/hygiene education in the developing world and adds a new dimension to MHM research. Investigation of the effectiveness of incorporating more physical objects in (menstrual) health education interventions is yet to be empirically investigated in terms of more long-term health-related behavioral outcomes. Thus, future research may investigate the effectiveness of this feminine hygiene kit, including analysis of its actual use and adoption. Furthermore, a randomized control trial study, with follow-up, of an educational intervention that incorporates hygiene/ sanitation experiential components in developing world contexts could also serve to further this line of research and provide further validation of our findings. Additionally, future examinations of WTP for this kit will consider including additional survey questions that enable the researcher to better determine consequentiality. This can be accomplished through eliciting perceptions on the outcomes of the survey and the methods of measuring preferences [63], or through priming the WTP question with mention of the policy impacts that can arise from the results of the survey [64].
Acknowledgements The authors would like to thank Christine Glidden, founder of Women2Be, for her generous time and cooperation and for providing the hygiene kits. They would also like to thank PratimanNeema Memorial Health Institute (PNMHI) and Paklihawa Vocational High School for organizing the events and helping in the implementation of the survey.

Author Contributions SKY contributed to the development of the research survey, and had primary responsibility for analyzing the data and drafting the manuscript. AKB contributed to the development of the survey and oversaw the editing and direction of the data analysis and manuscript development. SRC contributed to the development of the survey and editing of the manuscript. SKY is the overall guarantor.

Data Availability Statement The data generated and analyzed during this study are included in this published article (via its Supplementary Electronic Files).

\section{Compliance with Ethical Standards}

Funding No funding was received to carry out this study.

Conflict of interest Siobhan K. Yilmaz, Alok K. Bohara, and Soumi Roy Chowdhury have no conflicts of interest to declare.

Ethical Standards The study was carried out under the guidance of the Pratiman-Neema College to ensure that ethical standards consistent with those of the Declaration of Helsinki were maintained.

Informed Consent Informed consent was obtained from all individual participants in this study.

Open Access This article is distributed under the terms of the Creative Commons Attribution-NonCommercial 4.0 International License (http://creativecommons.org/licenses/by-nc/4.0/), which permits any noncommercial use, distribution, and reproduction in any medium, provided you give appropriate credit to the original author(s) and the source, provide a link to the Creative Commons license, and indicate if changes were made.

\section{Appendix}

Detailed regression results Tables 4, 5, 6 and 7 . 
Table 4 Log-linear regression of willingness to pay

\begin{tabular}{|c|c|c|c|c|c|}
\hline & Treatment Only & Log Model 1 & Log Model 2 & Log Model 3 & Log Model 4 \\
\hline \multirow[t]{2}{*}{ Treatment } & $0.199 * *$ & $0.158^{*}$ & 0.079 & 0.062 & 0.06 \\
\hline & $(0.066)$ & $(0.070)$ & $(0.087)$ & $(0.089)$ & $(0.088)$ \\
\hline \multirow[t]{2}{*}{ Respondent's Age } & & $0.017 * *$ & $0.017 * *$ & $0.017 * *$ & $0.016^{* *}$ \\
\hline & & $(0.005)$ & $(0.006)$ & $(0.006)$ & $(0.006)$ \\
\hline \multirow[t]{2}{*}{ Menstruation Age } & & -0.109 & -0.106 & -0.1 & -0.103 \\
\hline & & $(0.072)$ & $(0.073)$ & $(0.073)$ & $(0.075)$ \\
\hline \multirow[t]{2}{*}{ Reusable } & & $-0.295 * *$ & $-0.276^{* *}$ & $-0.282^{* *}$ & $-0.286 * *$ \\
\hline & & (0.099) & $(0.103)$ & $(0.104)$ & $(0.104)$ \\
\hline \multirow[t]{2}{*}{ Disposable } & & $-0.208 * *$ & $-0.214^{* *}$ & $-0.219^{* *}$ & $-0.228 * *$ \\
\hline & & $(0.070)$ & $(0.075)$ & $(0.074)$ & $(0.073)$ \\
\hline \multirow[t]{2}{*}{ School Env. Index } & & ! & 0.052 & 0.055 & 0.054 \\
\hline & & 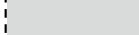 & $(0.037)$ & $(0.037)$ & $(0.037)$ \\
\hline \multirow[t]{2}{*}{ Cultural Norms Index } & & 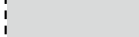 & -0.002 & 0.001 & -0.004 \\
\hline & & 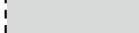 & $(0.032)$ & $(0.031)$ & $(0.033)$ \\
\hline \multirow[t]{2}{*}{ Wealth Index } & & 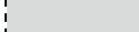 & 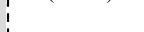 & 0.076 & 0.074 \\
\hline & & & & $(0.061)$ & $(0.063)$ \\
\hline \multirow[t]{2}{*}{ Buddhist } & & $\vdots$ & i & & -0.183 \\
\hline & & $\vdots$ & i & & $(0.279)$ \\
\hline Dalit & & 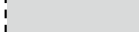 & i & & -0.043 \\
\hline \multirow{3}{*}{ Constant } & & & & & $(0.077)$ \\
\hline & $5.634 * * *$ & $5.746 * * *$ & $5.779 * * *$ & $5.657 * * *$ & $5.695 * * *$ \\
\hline & $(0.040)$ & $(0.183)$ & $(0.188)$ & $(0.206)$ & $(0.218)$ \\
\hline Observations & 169 & 169 & 169 & 169 & 169 \\
\hline $\ln (\mathrm{L})$ & -92 & -85 & -84 & -83 & -83 \\
\hline AIC & 187.1 & 182.8 & 184.4 & 184.4 & 187.6 \\
\hline $\mathrm{BIC}$ & 193.4 & 201.6 & 209.4 & 212.6 & 222.1 \\
\hline $\mathrm{R}^{2}$ & 0.053 & 0.119 & 0.131 & 0.141 & 0.145 \\
\hline
\end{tabular}

Robust standard errors are shown in parentheses

The dotted/shaded column represents the best-fit model based on the lowest AIC criterion

AIC Akaike Information Criterion, BIC Bayesian Information Criterion $* * * p<0.01, * * p<0.05, * p<0.1$

\begin{tabular}{|c|c|c|c|c|}
\hline & Log Model 1 & Log Model 2 & Log Model 3 & Log Model 4 \\
\hline \multirow[t]{2}{*}{ Treatment } & $0.249 * * *$ & $0.246^{* * *}$ & $0.229 * * *$ & $0.212 * *$ \\
\hline & $(0.064)$ & $(0.061)$ & $(0.067)$ & $(0.064)$ \\
\hline \multirow[t]{2}{*}{ Respondent's Age } & $0.009^{*}$ & $0.011 * *$ & $0.011 * *$ & $0.009^{*}$ \\
\hline & $(0.004)$ & $(0.004)$ & $(0.004)$ & $(0.004)$ \\
\hline \multirow[t]{2}{*}{ Menstruation Age } & -0.081 & -0.081 & -0.081 & -0.089 \\
\hline & $(0.069)$ & $(0.073)$ & $(0.073)$ & $(0.076)$ \\
\hline \multirow[t]{2}{*}{ Reusable } & $-0.216^{*}$ & $-0.206^{*}$ & $-0.209^{*}$ & $-0.219^{*}$ \\
\hline & ( & $(0.099)$ & $(0.101)$ & $(0.098)$ \\
\hline \multirow[t]{2}{*}{ Disposable } & $-0.258 * * *$ & $-0.223 * *$ & $-0.232 * *$ & $-0.256^{* *}$ \\
\hline & $(0.068)$ & $(0.082)$ & $(0.081)$ & $(0.079)$ \\
\hline \multirow[t]{2}{*}{ School Environment Index } & i & -0.025 & -0.022 & -0.021 \\
\hline & i & $(0.032)$ & $(0.032)$ & $(0.031)$ \\
\hline \multirow[t]{2}{*}{ Cultural Norms Index } & i & 0.006 & 0.003 & -0.009 \\
\hline & 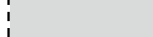 & $(0.033)$ & $(0.035)$ & $(0.034)$ \\
\hline \multirow[t]{2}{*}{ Wealth Index } & 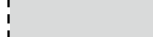 & , & 0.068 & 0.064 \\
\hline & & 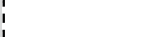 & $(0.063)$ & $(0.061)$ \\
\hline \multirow[t]{2}{*}{ Buddhist } & i & ' & & -0.332 \\
\hline & i & ' & & $(0.319)$ \\
\hline \multirow[t]{2}{*}{ Dalit } & i & i & & $-0.174^{*}$ \\
\hline & & & & $(0.073)$ \\
\hline \multirow[t]{2}{*}{ Constant } & $5.857 * * *$ & $5.799 * * *$ & $5.688 * * *$ & $5.816^{* * *}$ \\
\hline & $(0.175)$ & $(0.199)$ & $(0.221)$ & $(0.228)$ \\
\hline Observations & 169 & 169 & 169 & 169 \\
\hline $\ln (\mathrm{L})$ & -65 & -64 & -63 & -61 \\
\hline AIC & 141.4 & 144.2 & 144.7 & 143.1 \\
\hline $\mathrm{BIC}$ & 160.2 & 169.2 & 172.9 & 177.6 \\
\hline $\mathrm{R}^{2}$ & 0.242 & 0.247 & 0.253 & 0.278 \\
\hline
\end{tabular}

Robust standard errors are shown in parentheses

The dotted/shaded column represents the best-fit model based on the lowest AIC criterion

AIC Akaike Information Criterion, BIC Bayesian Information Criterion $* * * p<0.01, * * p<0.05, * p<0.1$ 
Table 6 GLM (gamma with log-link) regression of willingness to pay

\begin{tabular}{|c|c|c|c|c|}
\hline & GLM Model 1 & GLM Model 2 & GLM Model 3 & GLM Model 4 \\
\hline \multirow[t]{2}{*}{ Treatment } & $0.187 * *$ & 0.124 & 0.114 & 0.11 \\
\hline & $(0.070)$ & $(0.087)$ & $(0.087)$ & $(0.086)$ \\
\hline \multirow[t]{2}{*}{ Respondent's Age } & $0.015^{* *}$ & $0.015 * *$ & $0.015 * *$ & $0.013^{*}$ \\
\hline & $(0.005)$ & $(0.005)$ & $(0.005)$ & $(0.006)$ \\
\hline \multirow[t]{2}{*}{ Menstruation Age } & -0.137 & -0.124 & -0.119 & -0.119 \\
\hline & $(0.070)$ & $(0.071)$ & $(0.071)$ & $(0.072)$ \\
\hline \multirow[t]{2}{*}{ Reusable } & $-0.255^{* *}$ & $-0.234 *$ & $-0.235^{*}$ & $-0.238^{*}$ \\
\hline & $(0.094)$ & $(0.098)$ & $(0.098)$ & $(0.097)$ \\
\hline \multirow[t]{2}{*}{ Disposable } & $-0.160 *$ & $-0.167 *$ & $-0.169 *$ & $-0.186^{* *}$ \\
\hline & $(0.064)$ & $(0.067)$ & $(0.066)$ & $(0.065)$ \\
\hline \multirow[t]{2}{*}{ School Environment Index } & $\vdots$ & 0.047 & 0.046 & 0.045 \\
\hline & & $(0.034)$ & $(0.034)$ & $(0.034)$ \\
\hline \multirow[t]{2}{*}{ Cultural Norms Index } & $\begin{array}{l}\vdots \\
\vdots \\
\vdots\end{array}$ & 0.008 & 0.01 & 0.003 \\
\hline & ' & $(0.029)$ & $(0.029)$ & $(0.030)$ \\
\hline \multirow[t]{2}{*}{ Wealth Index } & $\begin{array}{l}\vdots \\
\vdots\end{array}$ & 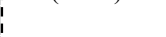 & 0.056 & 0.049 \\
\hline & 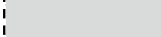 & 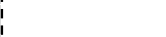 & $(0.059)$ & $(0.059)$ \\
\hline \multirow[t]{2}{*}{ Buddhist } & 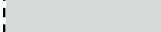 & 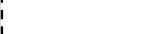 & & -0.154 \\
\hline & ' & i & & $(0.255)$ \\
\hline \multirow[t]{2}{*}{ Dalit } & i & ' & & -0.077 \\
\hline & & & & $(0.078)$ \\
\hline \multirow[t]{2}{*}{ Constant } & $5.859 * * *$ & $5.865 * * *$ & $5.771 * * *$ & $5.834 * * *$ \\
\hline & $(0.166)$ & $(0.169)$ & $(0.184)$ & $(0.187)$ \\
\hline Observations & 169 & 169 & 169 & 169 \\
\hline $\ln (\mathrm{L})$ & -1149 & -1149 & -1149 & -1149 \\
\hline$\chi^{2}$ & 29.4 & 30.2 & 30.7 & 34.4 \\
\hline AIC & 2309.8 & 2313.5 & 2315.3 & 2319.1 \\
\hline $\mathrm{BIC}$ & 2328.5 & 2338.5 & 2343.5 & 2353.6 \\
\hline $\mathrm{R}^{2 \mathrm{~d}}$ & 0.00162 & 0.00175 & 0.00181 & 0.00189 \\
\hline
\end{tabular}

Robust standard errors are shown in parentheses

The dotted/shaded column represents the best-fit model based on the lowest AIC criterion

AIC Akaike Information Criterion, BIC Bayesian Information Criterion, GLM generalized linear model

$* * * p<0.01, * * p<0.05, * p<0.1$

${ }^{a}$ GLM regression does not produce a regular $R^{2}$; calculated as McFadden's Pseudo $R^{2}=\left(1-\mathrm{LL}_{\mathrm{M}} / \mathrm{LL}_{0}\right)$ 
Table 7 GLM (gamma with log-link) regression of willingness to pay (w/IPW)

\begin{tabular}{|c|c|c|c|c|}
\hline & GLM Model 1 & GLM Model 2 & GLM Model 3 & GLM Model 4 \\
\hline Treatment & $\begin{array}{c}0.258 * * * \\
(0.062)\end{array}$ & $\begin{array}{c}0.254 * * * \\
(0.059)\end{array}$ & $\begin{array}{c}0.241 * * * \\
(0.065)\end{array}$ & $\begin{array}{c}0.219 * * * \\
(0.062)\end{array}$ \\
\hline Respondent's Age & $\begin{array}{l}0.008^{*} \\
(0.003)\end{array}$ & $\begin{array}{l}0.009 * \\
(0.004)\end{array}$ & $\begin{array}{c}0.010 * * \\
(0.004)\end{array}$ & $\begin{array}{c}0.007 \\
(0.004)\end{array}$ \\
\hline Menstruation Age & $\begin{array}{c}-0.108 \\
(0.066)\end{array}$ & $\begin{array}{c}-0.109 \\
(0.069)\end{array}$ & $\begin{array}{r}-0.109 \\
(0.069)\end{array}$ & $\begin{array}{c}-0.114 \\
(0.071)\end{array}$ \\
\hline Reusable & $\begin{array}{l}-0.169 \\
(0.088)\end{array}$ & $\begin{array}{c}-0.163 \\
(0.093)\end{array}$ & $\begin{array}{r}-0.163 \\
(0.094)\end{array}$ & $\begin{array}{c}-0.175 \\
(0.090)\end{array}$ \\
\hline Disposable & $\begin{array}{c}-0.194 * * \\
(0.061)\end{array}$ & $\begin{array}{r}-0.164^{*} \\
(0.070)\end{array}$ & $\begin{array}{r}-0.172 * \\
(0.069)\end{array}$ & $\begin{array}{c}-0.203^{* *} \\
(0.068)\end{array}$ \\
\hline School Environment Index & $\begin{array}{l}\vdots \\
\vdots \\
\vdots\end{array}$ & $\begin{array}{l}-0.02 \\
(0.028)\end{array}$ & $\begin{array}{c}-0.019 \\
(0.028)\end{array}$ & $\begin{array}{l}-0.018 \\
(0.027)\end{array}$ \\
\hline Cultural Norms Index & : & $\begin{array}{c}0.005 \\
(0.031)\end{array}$ & $\begin{array}{c}0.002 \\
(0.033)\end{array}$ & $\begin{array}{l}-0.01 \\
(0.032)\end{array}$ \\
\hline Wealth Index & $\begin{array}{l}\vdots \\
\vdots \\
1\end{array}$ & & $\begin{array}{c}0.057 \\
(0.060)\end{array}$ & $\begin{array}{c}0.049 \\
(0.057)\end{array}$ \\
\hline Buddhist & : & 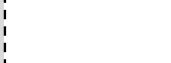 & & $\begin{array}{c}-0.3 \\
(0.272)\end{array}$ \\
\hline Dalit & : & & & $\begin{array}{c}-0.212 * * \\
(0.077)\end{array}$ \\
\hline Constant & $\begin{array}{c}5.945 * * * \\
(0.153)\end{array}$ & $\begin{array}{c}5.899 * * * \\
(0.179)\end{array}$ & $\begin{array}{c}5.802 * * * \\
(0.192)\end{array}$ & $\begin{array}{c}5.954 * * * \\
(0.196)\end{array}$ \\
\hline Observations & 169 & 169 & 169 & 169 \\
\hline $\ln (\mathrm{L})$ & -2345 & -2345 & -2345 & -2344 \\
\hline$\chi^{2}$ & 48.5 & 61.4 & 77 & 127.4 \\
\hline AIC & 4701.6 & 4705.4 & 4707.1 & 4709.6 \\
\hline $\mathrm{BIC}$ & 4720.4 & 4730.4 & 4735.3 & 4744 \\
\hline $\mathrm{R}^{2 \mathrm{~d}}$ & 0.00236 & 0.0024 & 0.00246 & 0.00279 \\
\hline
\end{tabular}

Robust standard errors are shown in parentheses

The dotted/shaded column represents the best-fit model based on the lowest AIC criterion

AIC Akaike Information Criterion, BIC Bayesian Information Criterion, GLM generalized linear model, $I P W$ inverse probability weighting

$* * * p<0.01, * * p<0.05, * p<0.1$

${ }^{a}$ GLM regression does not produce a regular $R^{2}$; calculated as McFadden's Pseudo $R^{2}=\left(1-\mathrm{LL}_{\mathrm{M}} / \mathrm{LL}_{0}\right)$

\section{References}

1. Sommer M, Sahin M. Overcoming the taboo: advancing the global agenda for menstrual hygiene management for schoolgirls. Am J Public Health. 2013;103(9):1556-9.

2. PSI. Menstrual Hygiene Management: a review of the evidence. Washington, D.C.; 2016.

3. House S, Mahon T, Cavill S. Bookshelf: Menstrual hygiene matters: a resource for improving menstrual hygiene around the world. Reprod Health Matters. 2013;21(41):257-9.

4. Anand E, Singh J, Unisa S. Menstrual hygiene practices and its association with reproductive tract infections and abnormal vaginal discharge among women in India. Sex Reprod Healthc. 2015;6(4):249-54.

5. World Health Organization. Health for the world's adolescents: a second chance in the second decade. World Health Organization; 2014 [cited 15 Feb 2016]. http://apps.who.int/adolescent/secon d-decade/\#.

6. Sinha K. 70\% Can't Afford Sanitary Napkins, Reveals Study. Times of India. 23 Jan 2011. http://timesofindia.indiatimes.com/ india/70-cant-afford-sanitary-napkins-reveals-study/articlesho w/7344998.cms? referral=PM.

7. Ndlovu E, Bhala E. Menstrual hygiene-a salient hazard in rural schools: a case of Masvingo district of Zimbabwe. Jamba. 2016;8(2):204.

8. Sommer M, Vasquez E, Worthington N, Sahin M. WASH in Schools Empowers Girls' Education: Proceedings of the
Menstrual Hygiene Management in Schools Virtual Conference 2012. UNICEF; 2012.

9. Auemaneekul N, Bhandari S, Kerdmongkol P. Menstrual hygiene practices among adolescents in a rural district of Nepal. Asia $\mathbf{J}$ Public Health. 2013;4:8-15.

10. UNESCO. Puberty Education and Menstrual Hygiene Management. Paris; 2014.

11. Sommer M, Caruso BA, Sahin M, Calderon T, Cavill S, Mahon T, et al. A time for global action: addressing girls' menstrual hygiene management needs in schools. PLOS Med. 2016;13(2):e1001962.

12. McMahon SA, Winch PJ, Caruso BA, Obure AF, Ogutu EA, Ochari IA, et al. "The girl with her period is the one to hang her head" reflections on menstrual management among schoolgirls in rural Kenya. BMC Int Health Hum Rights. 2011;11(1):7.

13. Haque SE, Rahman M, Itsuko K, Mutahara M, Sakisaka K. The effect of a school-based educational intervention on menstrual health: an intervention study among adolescent girls in Bangladesh. BMJ Open. 2014;4(7):e004607.

14. Jewitt S, Ryley H. It's a girl thing: menstruation, school attendance, spatial mobility and wider gender inequalities in Kenya. Geoforum. 2014;56:137-47.

15. Crofts T, Fisher J. Menstrual hygiene in Uganda schools: an investigation of low-cost sanitary pads. J Water Sanit Hyg Dev. 2012;2(1):50-8.

16. Hennegan J, Montgomery P. Do menstrual hygiene management interventions improve education and psychosocial outcomes for women and girls in low and middle income countries? A systematic review. PLoS One. 2016;11(2):e0146985. 
17. Shah SP, Nair R, Shah PP, Modi DK, Desai SA, Desai L. Improving quality of life with new menstrual hygiene practices among adolescent tribal girls in rural Gujarat, India. Reprod Health Matters. 2013;21(41):205-13.

18. Oster E, Thornton R. Menstruation, sanitary products, and school attendance: evidence from a randomized evaluation. Am Econ $\mathrm{J}$ Appl Econ. 2011;3(1):91-100.

19. Oster E, Thornton R. Menstruation and education in Nepal. 2009. http://www.nber.org/papers/w14853.

20. Wilson EF, Reeve JMK, Pitt AH, Sully BG, Julious SA. Investigating a reusable sanitary pad intervention in a rural educational setting-evaluating the acceptability and short term effect of teaching Kenyan school girls to make reusable sanitary towels on absenteeism and other daily activities: a partial preference parallel group, cluster randomized control trial. ScHARR Report Series. Report No.: 27. Sheffield: School of Health and Related Research, University of Sheffield; 2014.

21. Adukia A. Sanitation and education. Am Econ J Appl Econ. 2017;9(2):23-59.

22. Mbizvo M, Kasule J, Gupta V, Rusakaniko S, Kinoti S, MpanjuShumbushu W, et al. Effects of a randomized health education intervention on aspects of reproductive health knowledge and reported behaviour among adolescents in Zimbabwe. Soc Sci Med. 1997;44(5):573-7.

23. Fakhri M, Hamzehgardeshi Z, Hajikhani Golchin NA, Komili A. Promoting menstrual health among persian adolescent girls from low socioeconomic backgrounds: a quasi-experimental study. BMC Public Health. 2012;12(1):193.

24. Sommer M. An early window of opportunity for promoting girls health: policy implications of the girls puberty book project in Tanzania. Glob J Health Educ Promot. 2011;14(1):77-92.

25. Kuhlmann AS, Henry K, Wall LL. Menstrual hygiene management in resource-poor countries. Obstet Gynecol Surv. 2017;72(6):356-76.

26. Singh S, Bankole A, Woog V. Evaluating the need for sex education in developing countries: sexual behaviour, knowledge of preventing sexually transmitted infections/HIV and unplanned pregnancy. Sex Educ. 2005;5(4):307-31.

27. Sharma N, Sharma P, Sharma N, Wavare RR, Gautam B, Sharma $M$. A cross sectional study of knowledge, attitude, and practices of menstrual hygiene among medical students in north India. J Phytopharm. 2013;2(5):28-37.

28. BRIDGE. Gender update: Gender and Menstrual Hygiene Management (MHM). Bridge- Development and Gender. May 2015 (110) [cited 18 Oct 2016]. http://www.bridge.ids.ac.uk/updates/ gender-and-menstrual-hygiene-management-mhm.

29. WaterAid. Is menstrual hygiene and management an issue for adolescent school girls - a comparative study of four schools in different settings of Nepal. WaterAid; 2009. pp. 1-32. http://www. wateraid.org/nepal.

30. Adhikari P, Kadel B, Dhungel S, Mandal A. Knowledge and practice regarding menstrual hygiene in rural adolescent girls of Nepal. Kathmandu Univ Med J. 2007;3(19):382-6.

31. Dongre AR, Deshmukh PR, Garg BS. The effect of communitybased health education intervention on management of menstrual hygiene among rural Indian adolescent girls. World Health Popul. 2007;9(3):48-54.

32. Seraj KFB. Willingness to pay for improved sanitation services and its implications on demand responsive approach to BRAC water, sanitation, and hygiene programme. BRAC Research and Evaluation Division. Feb 2008; Working Paper. http://www.ircwa sh.org/sites/default/files/document1682008530.498501.pdf.

33. Terris-Prestholt F, Kumaranayake L, Macphail C, Rees H, Watts C. Distributing barrier methods for women: determinants of willingness to pay for microbicides, the diaphragm and the female condom. DFID SRH \& HIV RPC. Toronto; 2006. https://asset s.publishing.service.gov.uk/media/57a08c2440f0b64974001010/ MOPDC06.pdf.

34. Foreit JR, Foreit KGF. The reliability and validity of willingness to pay surveys for reproductive health pricing decisions in developing countries. Health Policy. 2003;63(1):37-47.

35. Rescorla RA. Pavlovian conditioning: it's not what you think it is. Am Psychol. 1988;43(3):151-60.

36. Kahneman D. A psychological perspective on economics. Am Econ Rev. 2003;93(2):162-8.

37. Baddeley M. Herding, social influence and economic decisionmaking: socio-psychological and neuroscientific analyses. Philos Trans R Soc B Biol Sci. 2010;365(1538):281-90.

38. Bushong B, King LM, Camerer CC, Rangel A. Pavlovian processes in consumer choice: the physical presence of a good increases willingness-to-pay. Am Econ Rev. 2010;100(4):1556-71.

39. Müller H. The real-exposure effect revisited-how purchase rates vary under pictorial vs. real item presentations when consumers are allowed to use their tactile sense. Int J Res Mark. 2013;30(3):304-7.

40. Reb J, Connolly T. Possession, feelings of ownership, and the endowment effect. Judgement Decis Mak. 2007;2(2):107-14.

41. Ericson KMM, Fuster A. The endowment effect. Cambridge, MA: National Bureau of Economic Research; 2013 Aug [cited 12 Feb 2018]. Report No.: w19384. http://www.nber.org/papers/w1938 4.pdf.

42. Wolf JR, Arkes HR, Muhanna W. The power of touch: an examination of the effect of duration of physical contact on the valuation of objects. Judgement Decis Mak. 2008;3(6):476-82.

43. Hermalin BE, Isen AM. A model of the effect of affect on economic decision making. Quant Mark Econ. 2008;6(1):17-40.

44. Romer PM. Thinking and feeling. Am Econ Rev. 2000;90(2):439-43.

45. Akaike H. A new look at the statistical model identification. IEEE Trans Autom Control. 1974;19(6):716-23.

46. Snow JC, Skiba RM, Coleman TL, Berryhill ME. Real-world objects are more memorable than photographs of objects. Front Hum Neurosci. 2014;8:837.

47. Gerhard TM, Culham JC, Schwarzer G. Distinct visual processing of real objects and pictures of those objects in 7- to 9-month-old infants. Front Psychol. 2016;7:827.

48. Domjan M. Pavlovian conditioning: a functional perspective. Annu Rev Psychol. 2005;56(1):179-206.

49. Dubé L, Bechara A, Böckenholt U, Ansari A, Dagher A, Daniel $\mathrm{M}$, et al. Towards a brain-to-society systems model of individual choice. Mark Lett. 2008;19(3-4):323-36.

50. Camerer C, Mobbs D. Differences in behavior and brain activity during hypothetical and real choices. Trends Cogn Sci. 2017;21(1):46-56.

51. Brasel SA, Gips J. Tablets, touchscreens, and touchpads: how varying touch interfaces trigger psychological ownership and endowment. J Consum Psychol. 2014;24(2):226-33.

52. Battjes-Fries MCE, Haveman-Nies A, Zeinstra GG, van Dongen EJI, Meester HJ, van den Top-Pullen R, et al. Effectiveness of taste lessons with and without additional experiential learning activities on children's willingness to taste vegetables. Appetite. 2017;109:201-8.

53. Angelopoulou MV, Kavvadia K, Taoufik K, Oulis CJ. Comparative clinical study testing the effectiveness of school based oral health education using experiential learning or traditional lecturing in 10 year-old children. BMC Oral Health. 2015;15(1):51.

54. Angelopoulou MV, Kavvadia K. Experiential learning in oral health education. J Educ Health Promot. 2018;7:70.

55. Hersch D, Perdue L, Ambroz T, Boucher JL. The impact of cooking classes on food-related preferences, attitudes, and behaviors of school-aged children: a systematic review of the evidence, 2003-2014. Prev Chronic Dis. 2014;11:E193. 
56. Ponce-Gonzalez I, Cheadle A, Aisenberg G, Cantrell LF. Improving oral health in migrant and underserved populations: evaluation of an interactive, community-based oral health education program in Washington state. BMC Oral Health. 2019;19(1):30.

57. Medic N, Ziauddeen H, Forwood SE, Davies KM, Ahern AL, Jebb SA, et al. The presence of real food usurps hypothetical health value judgment in overweight people. eNeuro. 2016;3(2).

58. Verhoeven AAC, Watson P, de Wit S. Failing to pay heed to health warnings in a food-associated environment. Appetite. 2018;120:616-26.

59. Carson RT, Groves T. Incentive and informational properties of preference questions. Environ Resour Econ. 2007;37(1):181-210.

60. Cummings RG, Taylor LO. Does realism matter in contingent valuation surveys? Land Econ. 1998;74(2):203.
61. List JA. Do explicit warnings eliminate the hypothetical bias in elicitation procedures? Evidence from field auctions for sportscards. Am Econ Rev. 2001;91(5):1498-507.

62. Cummings RG, Taylor LO. Unbiased value estimates for environmental goods: a cheap talk design for the contingent valuation method. Am Econ Rev. 1999;89(3):649-65.

63. Nepal M, Berrens R, Bohara A. Assessing perceived consequentiality: evidence from a contingent valuation survey on Global climate change. Int J Ecol Econ Stat. 2009;14(9):14-29.

64. Bulte E, Gerking S, List JA, de Zeeuw A. The effect of varying the causes of environmental problems on stated WTP values: evidence from a field study. J Environ Econ Manag. 2005;49(2):330-42. 